\title{
Improvement Voltage Stability and Load Ability Enhancement by Continuation Power Flow and Bifurcation Theory
}

\author{
Navid Ghaffarzadeh ${ }^{\star}$, Haniyeh Marefatjo, Iman Soltani, Fateme Salahian \\ Faculty of Engineering and Technology \\ Imam Khomeini International University Qazvin, Iran \\ e-mail: ghaffarzadeh@ikiu.ac.ir, h.marefat@ikiu.ac.ir, i_soltani@ikiu.ac.ir, f.salahian@ikiu.ac.ir
}

\begin{abstract}
Power systems operation becomes more important as the load demand increases all over the world. This rapid increase in load demand forces power systems to operate near critical limits due to economic and environmental constraints. The objective in power systems operation is to serve energy with acceptable voltage and frequency to consumers at minimum cost. This paper studies the important power system phenomenon; voltage stability voltage stability is studies by using continuation power flow method and the effect of compensator, placement of generator and variation of line reactance on the voltage stability have been studied. Voltage collapse scenario is presented which can be a serious result of voltage instability and also the parameters that affected by voltage collapse are discussed. In analysingxdsed power system voltage stability, continuation power flow method is utilized which consists of successive load flows. This method is applied to a 14 bus sample test system and load-voltage curves for several buses are obtained. Simulation is done with PSAT in MATLAB. Continuation Power Flow was implemented using Newton-Raphson method. Simulation results show the proper performance of capacitor, variation of line reactance and placement of generator to improve voltage control on the lines and significantly increase the load ability margin of power systems.
\end{abstract}

Keywords: continuation power flow method, voltage stability, voltage collapse, capacitor, reactance, generator.

\section{Introduction}

In recent years, the increase in peak load demand and power transfers between utilities has elevated concerns about system voltage security [1]. Power systems operation becomes more important as the load demand increases all over the world. This rapid increase in load demand forces power systems to operate near critical limits due to economic and environmental constraints. The objective in power systems operation is to serve energy with acceptable voltage and frequency to consumers at minimum cost. Reliability and security are also important parameters for power systems and should be satisfied. By reliability, it is meant that the system has adequate reserves in the face of changing energy demand. By security, it is meant that upon occurrence of a contingency, the system could recover to its original state and supply the same quality service as before. All these objectives can be achieved by proper planning, operation and control of power generation and transmission systems. Since generation and transmission units have to be operated at critical limits voltage stability problems may occur in power system when there is an increase in load demand. Voltage instability is one of the main problems in power systems. In voltage stability problem some or all buses voltages decrease due to insufficient power delivered to loads. In case of voltage stability problems, serious blackouts may occur in a considerable part of a system [2]. This can cause severe social and economic problems. In fact, more than 50 cases of voltage instability or voltage collapse were reported all over the world between 1965 and 1996. For example, a voltage collapse in the North American Western Systems Coordinating Council system on July 2, 1996, resulted in service interruptions to more than 6 million people [2]. The most common methods used in voltage stability analysis are continuation power flow, point of collapse, minimum singular value and optimization methods. In this study, continuation power flow method, widely used in voltage stability analysis, is utilized in order to analyse voltage stability of power systems. In section (2) of this paper the concept of voltage stability phenomena is described. Voltage stability can be 
analysed by using bifurcation theory, so in section (3) we focus on bifurcation theory and in section (4), Continuation Power flow method is detailed. The Case Study and simulation and results are presented in section (5) in the other hand and effects of compensation, transmission line reactance and adding new generating units are presented by analyzing bus voltage profiles that show the relationship between power and voltage. Finally; conclusion is discussed in section (6).

\section{Voltage Stability}

Power system stability can be divided into two as voltage stability and rotor angle stability. Rotor angle stability is the ability of interconnected synchronous machines of a power system to remain in synchronism [3]. In this kind of stability, power-angle equations are handled since power output of a synchronous machine varies as its rotor oscillates. Voltage stability is the ability of a power system to maintain steady acceptable voltages at all buses in the system under normal operating conditions and after being subjected to a disturbance [3]. Voltage stability can be attained by sufficient generation and transmission of energy. Generation and transmission units have definite capacities that are peculiar to them. These limits should not be exceeded in a healthy power system. Voltage stability problem arises when the system is heavily loaded that causes to go beyond limitations of power system. A power system enters a state of voltage instability when a disturbance, increase in load demand power or change in system condition causes a progressive and uncontrollable decline in voltage. The main factor causing instability is the inability of the power system to meet the demand for reactive power [3].

\subsection{Factors Affecting Voltage Stability}

The main reason for voltage instability is the lack of sufficient reactive power in a system. Generator reactive power limits and reactive power requirements in transmission lines are the main causes of insufficient reactive power.

\subsubsection{Reactive Power Limits of Generators}

Synchronous generators are the main devices for voltage control and reactive power control in power systems. In voltage stability analysis active and reactive power capabilities of generators play an important role. The active power limits are due to the design of the turbine and the boiler. Therefore, active power limits are constant. Reactive power limits of generators are more complicated than active power limits. There are three different causes of reactive power limits that are; stator current, over-excitation current and under-excitation limits. The generator field current is limited by over-excitation limiter in order to avoid damage in field winding. In fact, reactive power limits are voltage dependent. However, in load flow programs they are taken to be constant in order to simplify analysis [4].

\subsubsection{Transmission Lines}

Transfer of active and reactive power is provided by transmission lines. Since transmission lines are generally long, transfer of reactive power over these lines is very difficult due to significant amount of reactive power requirement[1].

\subsection{Voltage Collapse [3]}

Voltage collapse is the process by which the sequence of events accompanying voltage instability leads to a low unacceptable voltage profile in a significant part of system. When a power system is subjected to a sudden increase of reactive power demand, the required demand is met by the reactive power reserves supplied from generators and compensation devices. Most of the time, this can be achieved since there are sufficient reserves. Sometimes, it is not possible to meet this rapid increase in demand due to combination of events and system conditions. Thus, voltage collapse and a major breakdown of part or all of the system may occur.

There are some countermeasures that can be taken against voltage instability. Automatic voltage regulators (AVRs), under-load tap changers (ULTCs) and compensation devices are common ways to keep bus voltage magnitude in acceptable ranges[3]. 


\section{Bifurcation Theory}

In this section, it is explained the results of research and at the same time is given the comprehensive discussion. Results can be presented in figures, graphs, tables and others that make the reader understand easily [2],[5]. The discussion can be made in several sub-chapters.

Bifurcation theory is used to describe changes in the qualitative structures of the phase portrait when certain system parameters change. Local bifurcations can be studied by analyzing the vector differential equations near the bifurcation equilibrium points. Voltage collapse in power systems can be predicted by identifying parameter values that lead to saddle-node bifurcations. In order to present the characteristic of bifurcation, Equation 1 is considered.

$$
F(x, \lambda)=\dot{x}=\lambda-x^{2}
$$

In differential Equation $1, \mathrm{x}$ is the state variable and $\lambda$ is a parameter. There is a point called equilibrium point where: $F\left(x_{0}, \lambda_{0}\right)=0$. For this value of $\lambda$ the linearization of $F(x, \lambda)$ is singular.

Figure 1 is obtained for $F(x, \lambda)$, as $\lambda$ changes. When $\lambda=0$ there is a saddle node point. For $\lambda<0$, there is no equilibrium whereas for $\lambda>0$ there are two equilibrium points as stable and unstable points $[5,6]$.

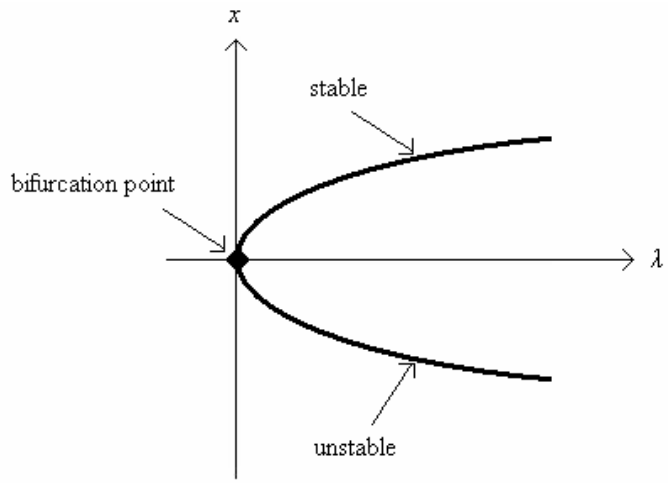

Figure 1. Bifurcation diagram for $f(x, \lambda)[7]$

\section{Continuation Power Flow}

The conventional power flow has a problem in the Jacobean matrix which becomes singular at the voltage stability limit. This problem can be overcome by using continuation power flow [8]. Figure 2 shows the predictor-corrector scheme used in the continuation power flow.

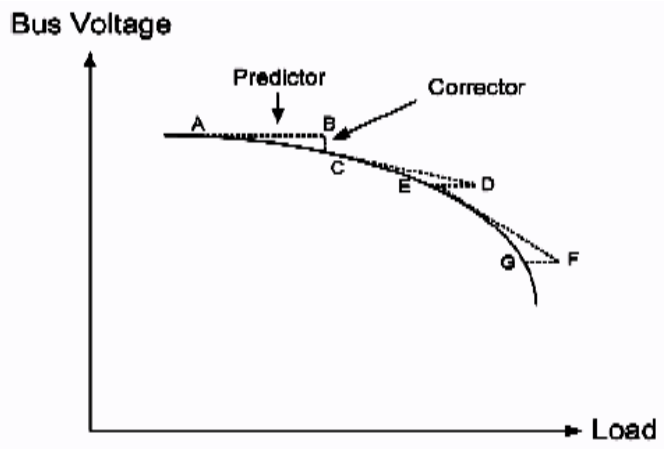

Figure. 2. The predictor-corrector scheme[9] 
From the Newton-Raphson, load flow equations can be written as:

$$
\begin{aligned}
& P_{i}-\sum_{j=1}^{N} Y_{i j} V_{i} V_{j} \cos \left(\delta_{i}-\delta_{j}-\theta_{i j}\right)=0 \\
& Q_{i}-\sum_{j=1}^{N} Y_{i j} V_{i} V_{j} \sin \left(\delta_{i}-\delta_{j}-\theta_{i j}\right)=0
\end{aligned}
$$

The new load flows equations consist of load factor are expressed as:

$$
\begin{aligned}
& P_{L i}=P_{L 0}+\lambda\left(K_{L i} S_{\Delta b a s e} \cos \emptyset_{i}\right) \\
& Q_{L i}=Q_{L 0}+\lambda\left(K_{L i} S_{\Delta b a s e} \sin \emptyset_{i}\right)
\end{aligned}
$$

where:

$P_{L 0}, Q_{L 0}=$ original load at bus $\mathrm{i}$, active and reactive power respectively

$K_{L i}=$ multiplier to designate the rate of load change at bus $\mathrm{i}$ as $\lambda$ changes

$S_{\Delta b a s e}=$ a given quantity of apparent power which is chosen to provide appropriate scaling of $\lambda$

The power flow equations can be written as:

$F(\delta, V, \lambda)=0$

Then the active power generation term can be modified to:

$P_{G i}=P_{G 0}\left(1+\lambda \mathrm{K}_{\mathrm{Gi}}\right)$

where:

$P_{G 0}=$ The initial value of active power generation

$P_{G i}=$ the active power generation at bus $\mathrm{i}$

$K G \mathrm{i}=$ the constant of changing rate in generation

To solve the problem, the continuation algorithm starts from a known solution and uses a predictor-corrector scheme to find subsequent solutions at different load levels [9].

\section{Case Study and Simulation}

In this test system, bus 1 is chosen as slack bus, bus 2 is voltage control bus and other buses are load buses. Sample test system consists of 3 areas those 14 buses, 2 generators, 20 transmission lines and 11 loads. The first area includes buses 1 to 5 and has a voltage level is $69 \mathrm{KV}$. The second area consists of 7 to 14 buses and has a voltage level is $13.8 \mathrm{KV}$. The third area consists bus 6 and has a voltage level is $18 \mathrm{KV}$. The single line diagram of 14-bus test system is shown in Figure 3. Continuation

power flow method is applied to sample test system using PSAT program and voltage profiles of 14 buses are obtained. Bus voltages are plotted with respect to the load parameter in Figure 4. As the load parameter is increased, bus voltages of load buses decrease as it is expected. The continuation power flow result given in table 1 and table 2 . 


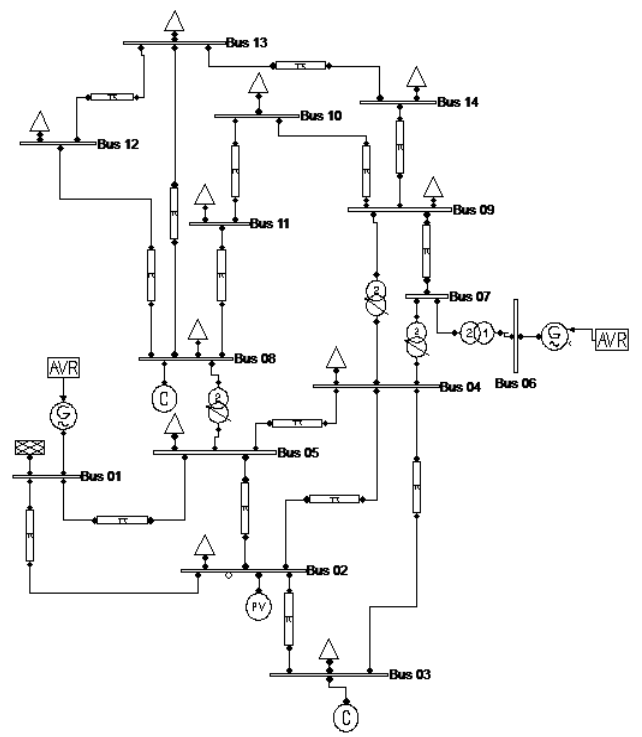

Figure. 3. Single line Diagram of 14-Bus

Table 1. Voltage magnitude and phase angle for 14-bus test system

\begin{tabular}{ccc}
\hline \multirow{2}{*}{ Bus } & $\begin{array}{c}\text { Continuation } \\
\text { Voltage }(\mathrm{Kv})\end{array}$ & $\begin{array}{c}\text { Power Flow } \\
\text { Angle(deg) }\end{array}$ \\
\hline 1 & 73.14 & 0 \\
2 & 72.105 & -36.1442 \\
3 & 69.69 & -84.607 \\
4 & 47.8139 & -69.5666 \\
5 & 46.7392 & -58.8404 \\
6 & 19.62 & -94.9406 \\
7 & 10.9344 & -94.9406 \\
8 & 14.766 & -110.3449 \\
9 & 9.6586 & -107.5489 \\
10 & 9.9929 & -110.1698 \\
11 & 12.1132 & -110.785 \\
12 & 13.4993 & -114.1077 \\
13 & 12.8266 & -114.012 \\
14 & 9.4878 & -118.4656 \\
\hline
\end{tabular}

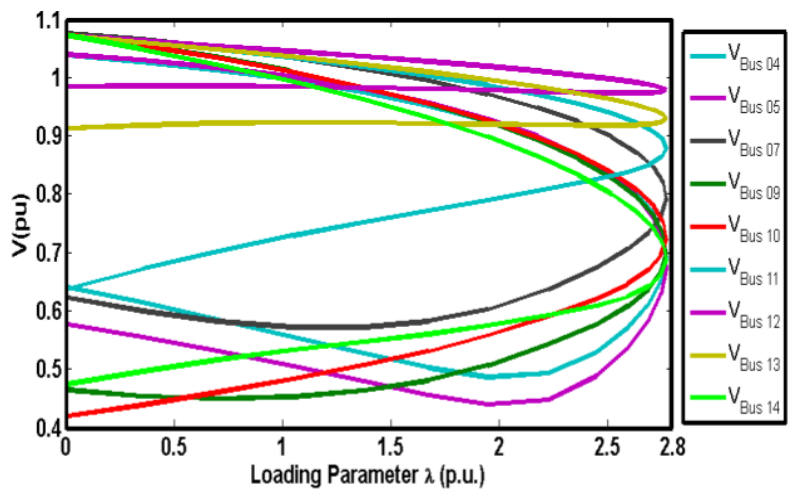

Figure. 4. Voltage profiles of 14-Bus Test System 
Table 2. Power flow for 14-bus test system

\begin{tabular}{cccc}
\hline Line & $\begin{array}{c}\text { From-To } \\
\text { bus }\end{array}$ & \multicolumn{2}{c}{ Continuation Power Flow } \\
$\mathrm{P}(\mathrm{pu})$ & $\mathrm{Q}(\mathrm{pu})$ \\
\hline 1 & $2-5$ & 2.1654 & 1.7968 \\
2 & $8-12$ & 0.37147 & 0.21428 \\
3 & $12-13$ & 0.11528 & 0.11117 \\
4 & $8-13$ & 0.86051 & 0.73302 \\
5 & $8-11$ & 0.43171 & 0.8281 \\
6 & $11-10$ & 0.22369 & 0.60682 \\
7 & $9-10$ & 0.17614 & -0.26066 \\
8 & $9-14$ & 0.30077 & -0.07715 \\
9 & $14-13$ & -0.3018 & -0.32418 \\
10 & $7-9$ & 1.1004 & 0.78744 \\
11 & $1-2$ & 11.1173 & 0.20086 \\
12 & $3-2$ & -3.4144 & 2.4056 \\
13 & $3-4$ & -0.23704 & 2.0877 \\
14 & $1-5$ & 3.3737 & 2.5271 \\
15 & $5-4$ & 1.8691 & 0.65127 \\
16 & $2-4$ & 2.8627 & 1.8018 \\
17 & $5-8$ & 2.0978 & 0.15225 \\
18 & $4-9$ & 0.52002 & 0.19735 \\
19 & $4-7$ & 1.1004 & 0.02388 \\
20 & $6-7$ & 0 & 1.8418 \\
\hline
\end{tabular}

When Figure 4 is examined it can be seen that the most reduction in bus voltages occurs in 14,9 and 10 buses. It can be concluded from this result that bus 14 is the weakest bus in this sample system. The bus with the highest voltage sensitivity factor can be thought as the weakest bus in a system. Weakest bus is more sensitive to load changes. In other words, the load connected to this bus is affected more than other loads in case of an unexpected load increase. Sample system loses its voltage stability at the critical point where the load parameter value is 2.7687 as seen in Figure 4. The critical point can be taken as voltage collapse point. System becomes voltage unstable beyond this point and voltage decreases rapidly due to requirement of reactive power in the system. In the next part, effect of line reactance, compensator and add the generator units on voltage stability are Study.

\subsection{Effect of Compensation on Voltage Stability}

In order to illustrate the effect of compensation in voltage stability, shunt capacitor banks ranging from 0.1 to $0.5 \mathrm{pu}$ in 0.1 pu steps are connected respectively to bus 14 (weakest bus) and continuation power flow is performed for all cases. It is expected to see the critical point at the highest loading level in capacitor bank with 0.5 pu case. Figure 5 shows the voltage profiles for base and other five cases of bus 14 obtained in continuation power flows. It is obviously seen that maximum loading point increases as compensation value increases.

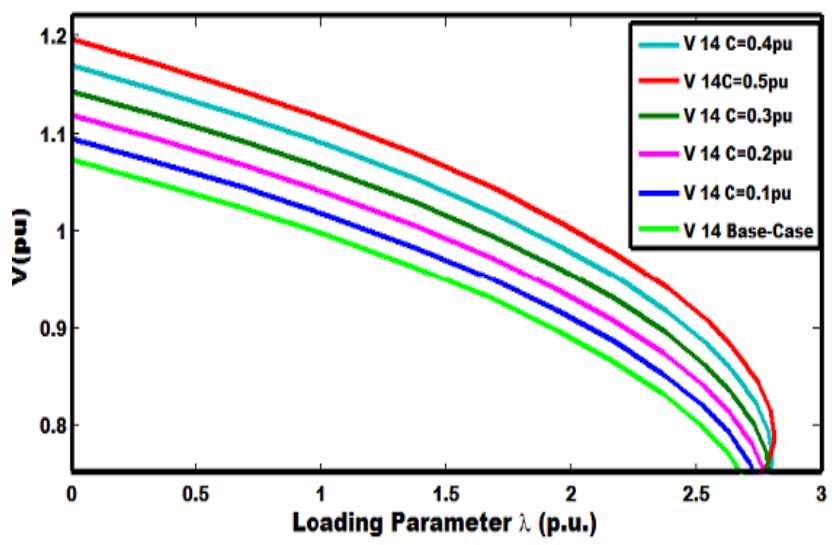

Figure 5. Voltage profiles of Bus 14 for different compensation cases (0.1pu-0.5pu) 
In the base case, load parameter is 2.7687 whereas in 0.5 pu shunt compensation case, it increases to 2.8149. Adding shunt capacitor to power system enhances the voltage stability limits. Therefore, for some situations it prevents voltage collapse. Adding a shunt capacitor to bus 14 improves the voltage stability limit not only in bus 14 but also in other buses. Table 3 shows the voltage at bus 14 and loading parameter for the all shunt capacitor cases.

Table 3. voltage at bus 14 and loading parameter for the all shunt capacitor cases

\begin{tabular}{lcc}
\hline & $\begin{array}{c}\text { Loading } \\
\text { Parameter } \\
\text { (p.u.) }\end{array}$ & $\begin{array}{c}\text { Volt bus } \\
14(\mathrm{KV})\end{array}$ \\
\hline Base-case & 2.7687 & 9.4878 \\
Cap=0.1pu & 2.7796 & 9.7635 \\
Cap=0.2pu & 2.7889 & 10.0286 \\
Cap=0.3pu & 2.798 & 10.3034 \\
Cap=0.4pu & 2.8067 & 10.5886 \\
Cap=0.5pu & 2.8149 & 10.8848 \\
\hline
\end{tabular}

When voltage in Table 3 compare with base-case, it is seen that voltages in bus 14 increase in all shunt capacitor cases which shows the enhancement in voltage stability

\subsection{Effect of New Generators on Voltage Stability}

After presenting the effect of compensation, transmission line reactance effect on voltage stability is presented by performing continuation Power flow for different line reactance values. In order to analyse the effect of transmission lines reactance, again the weakest bus in the system, bus 14 is observed by performing continuation power flows for different line reactance values between bus 13 and bus 14, X13-14. Similar to compensation cases analysis, five continuation power flows are done for $\mathrm{X} 13-14,0.8 \times 13-14,0.6 \times 13-14,0.4 \times 13-14$ and $0.2 \times 13-14$ and voltage profiles of bus 14 are observed for these cases. In these cases, it is expected to see a better voltage profile as line reactance decreases since transmission line reactance cause significant amount of reactive power requirement in systems Figure 6 shows the voltage profiles for different line reactance values for X13-14 which is the line reactance of transmission line between 13 and 14 buses. As it is seen in Figure 6, load parameter in critical point increases as line reactance $\mathrm{X} 13-14$ decreases. Load parameter for $0.2 \times 13-14$ case is approximately 2.7783 . It means that bus 14 lose its voltage stability after this critical point which is greater than the base case.

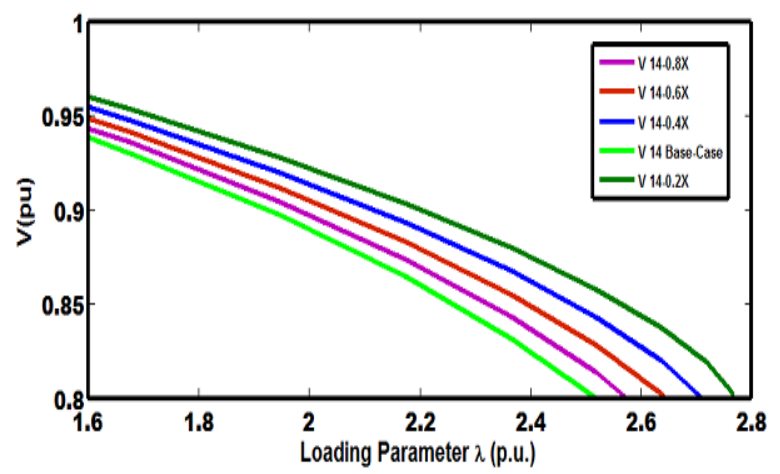

Figure 6. Voltage profiles of Bus 14 for different reactance cases between bus 13 and 14 
Table 4 shows the voltage at bus 14 and loading parameter for the all line reactance cases.

Table 4. voltage at bus 14 and loading parameter for the all line

\begin{tabular}{lcc}
\hline & Loading & Volt bus \\
& Parameter (p.u.) & $14(\mathrm{KV})$ \\
\hline Base-case & 2.7687 & 9.4878 \\
Reac $=0.8 \mathrm{X}$ & 2.773 & 9.8271 \\
Reac $=0.6 \mathrm{X}$ & 2.7762 & 10.1739 \\
Reac $=0.4 \mathrm{X}$ & 2.778 & 10.5292 \\
Reac $=0.2 \mathrm{X}$ & 2.7783 & 10.5292 \\
\hline
\end{tabular}

When voltage in table 4 compare with base-case it is seen that voltages in bus 14 increase in all line reactance cases which shows the enhancement in voltage stability.

\subsection{Effect of New Generators on Voltage Stability}

Lastly, three new generators to be connected to buses 2, 3 and 8 in sample system. Figure 7 shows the voltage profiles of bus 14 for the case three new generators and base case. When it is compared with Figure 4 it is easily seen that critical point moves to right in new case. Since reactive power generating limit of whole system increases by adding generators, system can keep bus voltages stable for higher loadings when compared with base case.

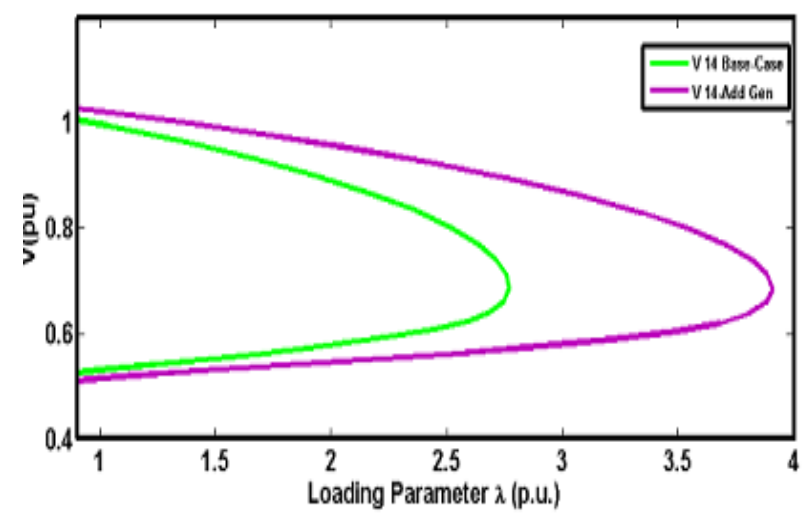

Figure. 7. Comparisons voltage profiles between the addition generator in to the system and the base case

Table 5. voltage at bus 14 and loading parameter for addition generators to system

\begin{tabular}{ccc}
\hline & $\begin{array}{c}\text { Loading Parameter } \\
\text { (p.u.) }\end{array}$ & Volt bus 14(KV) \\
\hline Base-case & 2.7687 & 9.4878 \\
Gen:2,3,8 & 3.906 & 9.7635 \\
\hline
\end{tabular}

As can be seen Lambda has increased the amount of 2.7687 to 3.906. Second area voltage for $\mathrm{C}=0.5 \mathrm{pu}, \mathrm{X}=0.2 \times 13-14$ and adding new generators presented in table 6 . Second area voltage average for $\mathrm{C}=0.5 \mathrm{pu}, \mathrm{X}=0.2 \times 13-14$ and adding new generators is given in table 7 and Percentage changes are calculated compared to the base case. 
Table 6. voltage at buses (7-14) for difference cases

\begin{tabular}{|c|c|c|c|c|}
\hline $\begin{array}{c}\text { Bus. } \\
\text { No }\end{array}$ & Base & & \multicolumn{2}{|c|}{ Voltage at bus $14(\mathrm{KV})$} \\
\hline 7 & 10.9344 & 11.1827 & 11.2519 & 10.9532 \\
\hline 8 & 14.766 & 14.766 & 14.766 & 14.766 \\
\hline 9 & 9.6586 & 10.1796 & 10.2375 & 9.6623 \\
\hline 10 & 9.9929 & 10.4361 & 10.4911 & 9.9915 \\
\hline 11 & 12.1132 & 12.3389 & 12.3747 & 12.1107 \\
\hline 12 & 13.4993 & 13.6588 & 13.4352 & 13.4914 \\
\hline 13 & 12.8266 & 13.1381 & 12.669 & 12.8155 \\
\hline 14 & 9.4878 & 10.8845 & 10.8922 & 9.4695 \\
\hline
\end{tabular}

As seen in the case of the capacitor $0.5 \mathrm{pu}$ is connected to the bus 14 , we have highest increase in voltage buses, while adding new generators to the system compare the base case does not cause significant changes in voltage buses.

Table 7. Voltages percentage changes compared to the base case for all buses

\begin{tabular}{ccccc}
\hline & Base & $\mathrm{C}=0.5 \mathrm{pu}$ & $\mathrm{X}=0.2 \mathrm{x}$ & Gen \\
\hline Ave. Vol(pu) & 0.84491 & 0.87486 & 0.87063 & 0.84474 \\
Per\% & - & 3.54 & 3.044 & -0.0201 \\
\hline
\end{tabular}

Lambda max and Percentage changes compared to the base case for all buses for $\mathrm{C}=0.5 \mathrm{pu}, \mathrm{X}=0.2 \times 13-14$ and adding new generators presented in table 8 .

As seen in the case of adding generator units to system, we have highest increase in lambda max, in this case Lambda has increased by $41 \%$ compared to the base case, and means improve the voltage stability of the network is compared to base case, while in case $\mathrm{X}=0.2 \times 13-14$ compare the base case does not cause significant changes in lambda max, lambda has increased by $0.3467 \%$ compared to the base case.

Table 8. lambda max Percentage changes compared to the base case for all buses

\begin{tabular}{ccccc}
\hline & Base & $\mathrm{C}=0.5 \mathrm{pu}$ & $\mathrm{X}=0.2 \mathrm{x}$ & Gen \\
\hline Lambda max & 2.7687 & 2.8149 & 2.7783 & 3.906 \\
Per\% & - & 1.6686 & 0.3467 & 41.077 \\
\hline
\end{tabular}

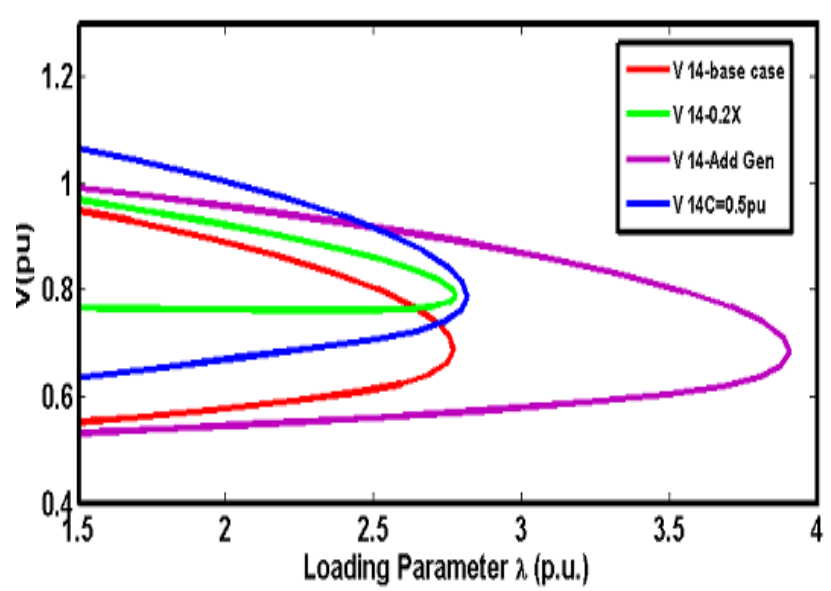

Figure 8. Comparisons voltage profiles between the addition generator in to the system, compensator and variation reactance and the base case 


\section{Conclusion}

In this paper, voltage stability phenomena and continuation power flow method, used in voltage stability analysis of power systems, are presented. The presented method is applied to 14-Bus sample test system. Voltage magnitude and bus voltage versus load parameter curves are obtained for several scenarios by using PSAT software, which is a one of the toolbox of MATLAB software. The effect of compensation is discussed by adding shunt capacitors in different per unit values to the bus defined in sample system. It is observed from voltage profiles and voltage magnitude that adding shunt capacitor to a bus cause to enhance the voltage stability of whole buses in sample system. Since the shunt capacitor injects reactive power to system. Thus, critical point occurs in higher loading levels and the magnitudes of bus voltages will be increased. In addition, the effect of variation of line reactance on voltage stability is studied by performing five continuation power flows to the proposed system. Voltage profiles for different line reactance cases prove the enhancement in voltage stability. With decreases of line reactance, reactive power demand decreases and profile of buses voltages is improved. Finally, effect of adding three new generating units is also observed in sample test system. Adding new generators improves the voltage stability of sample system since total power generation increases.

\section{References}

[1] V Ajjarapu and C Christy. The continuation power flow: A tool for steady state voltage stability analysis. IEEE Trans. on Power Systems, 1992; 7(1): 426-423.

[2] M Larsson. Coordinated Voltage Control in Electric Power Systems. Doctoral Dissertation. Lund University. 2000.

[3] P Kundur. Power System Stability and Control. McGraw-Hill. 1994.

[4] S Repo. On-line Voltage Stability Assesment of Power System, An Approach of Black-box Modelling. Tampere University of Technology Publications 344. 2001.

[5] WD Rosehart and CA Cañizares. Bifurcation Analysis of Various Power System Models. International Journal of Electrical Power \& Energy Systems. March 1999; 21(3): 171-182.

[6] A Cañizares. Voltage Collapse and Transient Energy Function Analyses of AC/DC Systems. Doctoral Dissertation. University of Wisconsin- Madison. 1991.

[7] Mehmet B Kesk_n. Continuation Power Flow and Voltage Stability in Power Systems. A Thesis Submitted to the Graduate School of Natural and Applied Sciences of Middle East Technical University. Sep2007.

[8] Venkataramana Ajjarapu, Colin Christy. The continuation power flow a tool for steady state voltage stability analysis. IEEE Transactions on Power Systems. 1992; 7(1).

[9] R Kalaivani, V Kamaraj. Modeling of Shunt FACTS Devices for Voltage Stability Enhancement. ISSN 1450-216X. 2011; 61(1): 144-154. 